\title{
Simple General Purpose Ion Beam Deceleration System Using a Single Electrode Lens
}

\author{
J. Lopes ${ }^{1}$, J. Rocha ${ }^{2}$ \\ ${ }^{1}$ Instituto Superior de Engenharia de Lisboa, Rua Conselheiro Emídio Navarro, Lisbon, Portugal \\ ${ }^{2}$ Instituto de Plasmas e Fusão Nuclear, Campus Tecnológico e Nuclear, Instituto Superior Técnico, Lisboa, \\ Portugal \\ Email: jgabriel@deea.isel.ipl.pt, jroc@ctn.ist.utl.pt
}

Received 28 June 2015; accepted 3 August 2015; published 6 August 2015

Copyright (C) 2015 by authors and Scientific Research Publishing Inc.

This work is licensed under the Creative Commons Attribution International License (CC BY). http://creativecommons.org/licenses/by/4.0/

(c) (i) Open Access

\begin{abstract}
Ion beam deceleration properties of a newly developed low-energy ion beam implantation system were studied. The objective of this system was to produce general purpose low-energy (5 to 15 $\mathrm{keV}$ ) implantations with high current beam of hundreds of $\mu \mathrm{A}$ level, providing the most wide implantation area possible and allowing continuously magnetic scanning of the beam over the sample(s). This paper describes the developed system installed in the high-current ion implanter at the Laboratory of Accelerators and Radiation Technologies of the Nuclear and Technological Campus, Sacavém, Portugal (CTN).
\end{abstract}

\section{Keywords}

\section{Deceleration, Low Energy, Positive Ion Beam}

\section{Introduction}

Low energy ion implantation became very important nowadays, either on microelectronics technology, thin film studies or plasma-wall studies in fusion reactors. Traditional ion deceleration uses a system that includes an electrostatic Einzel lens to focus and a target disk to decelerate the ion beam [1]-[3]. The objective of the described work was to develop a system to focus and decelerate a positive ion beam, keeping magnetic scanning of the beam possible. With this system the high-current ion implanter installed at the Nuclear and Technological Campus, can make implantation from less than 5 to $210 \mathrm{keV}$. 


\section{System Description}

To produce ion implantations at low energies it is necessary to decelerate the beam, keeping it focused. Positive beam deceleration is performed by applying a positive voltage to the target $\left(V_{A}\right)$, in order to create an axial electric field in the opposite direction of propagation of the ion beam $(E)$. This is the method (Figure 1) used in most of ion implanters.

The difficulty in the deceleration process is the creation of a uniform axial field in order to decelerate the beam without defocus. The most common method is to use Einzel lenses [4]-[6] to focus the beam in the lens axis. This method restricts the implantation area to a small region. In a general purpose system where multiple samples of different dimensions and large beam scanning has preference over a sharp focus, a single electrode lens can be more useful.

In order to correct the dispersion of the ion beam allowing magnetic scanning to run continuously it was necessary to study the behaviour of a positive ion beam in two aspects: in front of a charged target and in the region inside a charged ring.

This study, using simple electromagnetism theory, showed that a positive ion beam deflection of length $y_{i}$ due to a uniform electric field with opposite direction of the beam can be described by [7]-[9]

$$
y_{i}=-\sqrt{\frac{x}{K}+\frac{1}{4 \cdot K^{2} \cdot \tan ^{2} \alpha}}-\frac{1}{2 \cdot K \cdot \tan \alpha}
$$

where

$$
K=-\frac{E_{x}}{4 \times E c_{o} \times \operatorname{sen}^{2} \alpha}
$$

being $E c_{o}$ the initial beam energy and $\alpha$ the incident angle and $E_{x}$ the electric field on the $x$ direction, given by

$$
E_{x}=\frac{C_{a} \cdot V_{a}}{4 \cdot \pi \cdot R_{a}^{2} \cdot \varepsilon_{o}}\left(1-\frac{x}{\sqrt{R_{a}^{2}+x^{2}}}\right)
$$

where $V_{a}$ is the voltage applied to the target, $C_{a}$ the self-capacitance and $R_{a}$ the target radius.

As the field is non-uniform, the final deflection $y_{f}$ is given by the sum of elementary regions of uniform fields as in

$$
y_{f}=\sum_{1}^{n} y_{i}
$$

being $n$ the number of considered uniform regions.

On the other hand, the study of a charged ring showed that the electric field in the $y$ direction inside the ring, that forms the single electrode lens, can be given by

$$
E_{y}=E_{y o} \cdot \mathrm{e}^{\left(-x^{2} / 2 \cdot\left(k_{1} \cdot y+k_{2}\right)^{2}\right)}
$$

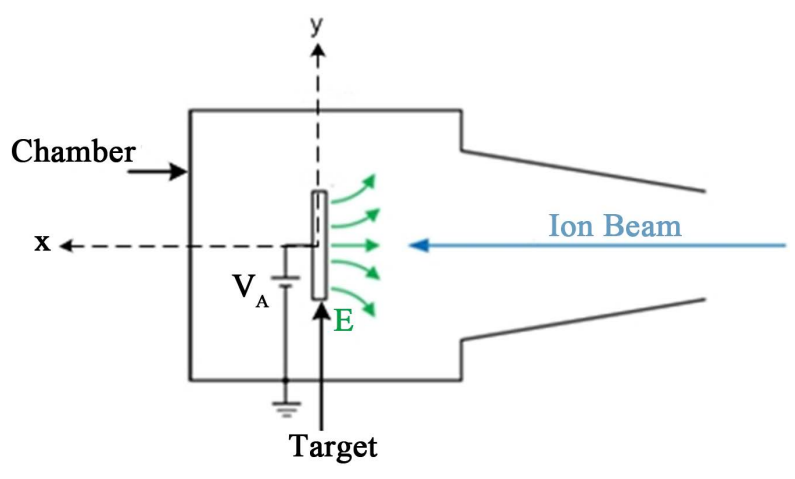

Figure 1. Deceleration method for a positive ion beam. 
where

$$
E_{y o}=\frac{l \cdot V}{2 \cdot R^{2} \ln \left(\frac{8 \cdot R}{r}\right)} \cdot k_{3} \cdot\left(\frac{1}{R+y}-\frac{1}{R-y}\right)
$$

being $V$ the voltage applied to the ring, $l$ the ring length, $R$ the ring radius, $r$ the electrode radius and $k_{1}, k_{2}$ and $k_{3}$ geometrical constants of the system.

Thus the beam deflection due to a charged ring is given by

$$
D_{y}=\frac{d \cdot E_{y o} \cdot \sqrt{2 \pi}}{2 \cdot E c_{o}} \cdot\left(k_{1} \cdot y+k_{2}\right)
$$

where $d$ is the distance between the target and the lens.

Thus, using Equations (4) and (7) was possible to determine the voltage to apply to the electrostatic lens to compensate for the deflection of the ion beam caused by the charged target. The theoretical approach was also useful to determine the best overall geometry of the system.

To confirm these results, the simulation software Simion 3D 8.0 was used to simulate the performance of the electrostatic lens, enabling to verify the best target and lens dimensions and gap in between to subsequently build the system [5].

In Figure 2 the non-charged target is showed, the lines in blue represent the various possible trajectories for a positive ion beam of $15 \mathrm{keV}$ energy.

In Figure 3 is represented one simulation with the same conditions but with a target potential of $10 \mathrm{kV}$ (beam energy of $5 \mathrm{keV}$ ). In this situation deflection exists, as predicted in the theoretical study. The red lines represent the electric field equipotentials.

Through this figure is also possible to verify that the deflection increases as the target is biased at higher potentials, as expected i.e. as final energy becomes smaller, the beam becomes defocused.

For subsequent simulations a lens was introduced. As shown in Figure 4, the simulation confirms the theoretical prediction that, using a charged lens with only one electrode with a given potential, it is possible, for most trajectories, to compensate the deflection caused by the target, relaying the path of the beam to its original position. It is also shown that the outmost trajectories are over-deflected.

Table 1 and Figure 5 shows the theoretical and simulated values for the potential applied to the lens $\left(V_{L}\right)$ for which the most significant trajectories are better compensated for various values of the target potential ( $\left.V_{A}\right)$, using the same geometry in both cases.

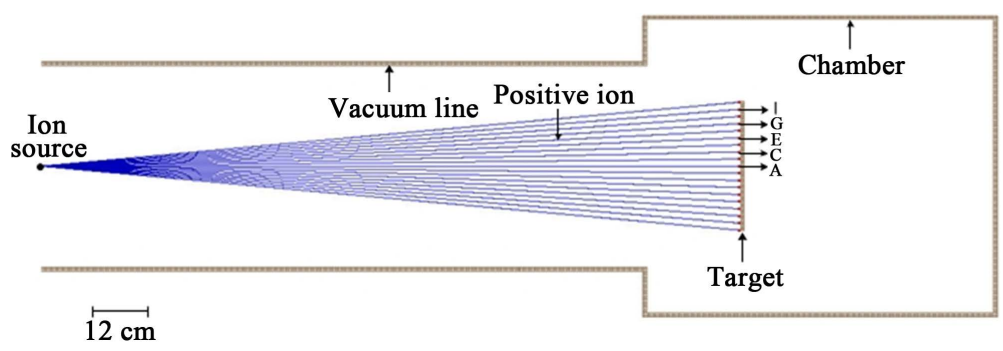

Figure 2. Ion beam simulation with a non-charged (grounded) target.

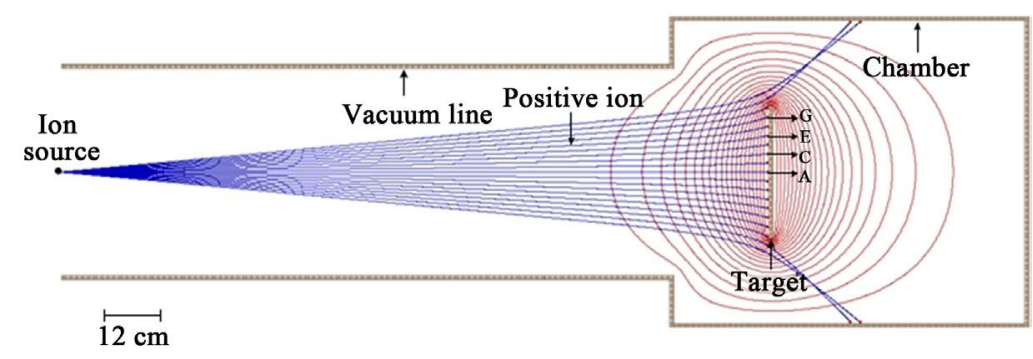

Figure 3. Ion beam simulation with target biased at $10 \mathrm{kV}$. 


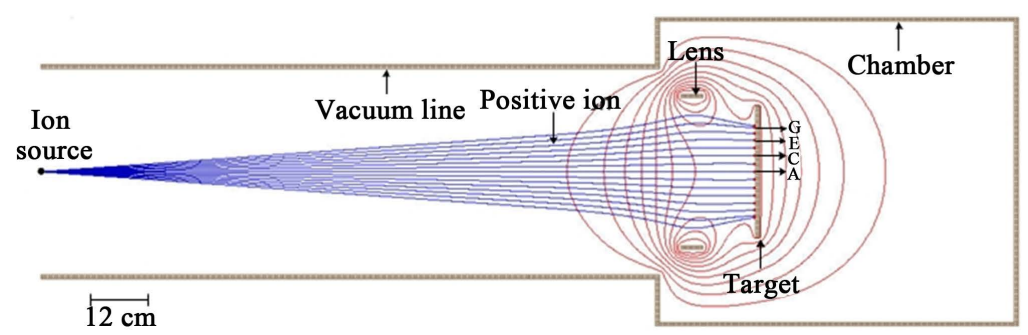

Figure 4. Ion beam simulation at $V_{A}=10 \mathrm{kV}$ and $V_{L}=15.5 \mathrm{kV}$.

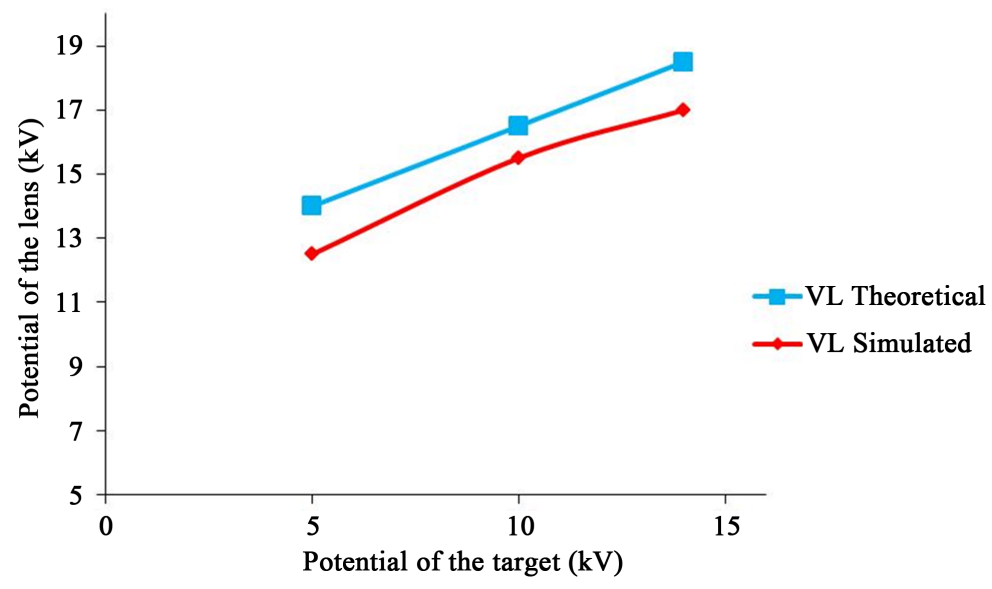

Figure 5. $V_{L}=f\left(V_{A}\right)$ theoretical and simulated.

Table 1. $V_{L}=f\left(V_{A}\right)$ theoretical and simulated.

\begin{tabular}{ccc}
\hline$V_{A}[\mathrm{kV}]$ & $V_{L}[\mathrm{kV}]$ Theoretical & $V_{L}[\mathrm{kV}]$ Simulated \\
\hline 5 & 14 & 12.5 \\
10 & 16.5 & 15.5 \\
14 & 18.5 & 17 \\
\hline
\end{tabular}

The equation for the deflection caused by the electric field (7), is in general terms in agreement with the simulations, as long it is applied to flat samples to avoid changes in the geometry of the target and consequently in the electric field.

\section{Experimental Results}

In Figure 6 is showed the circuit used for deceleration and focusing of the beam.

In this circuit, two high voltage $(20 \mathrm{kV})$ power supplies are used, one to bias the lens $\left(V_{L}\right)$ and the other to bias the target $\left(V_{A}\right)$. Resistors $\mathrm{R}_{1}$ and $\mathrm{R}_{2}$ are used to improve voltage stability, while the resistor $\mathrm{R}_{3}$ is intended to measure the beam current [10].

Three sets of tests were performed in order to proof homogeneity of implantations using the studied system. Each test consists of an implantation of four samples of silicon with an Argon beam with final energy of $5 \mathrm{keV}$. The implantation area was $10 \times 10 \mathrm{~cm}^{2}$ and beam current of $100 \mu \mathrm{A}$ (Figure 7). The first test consists of an implantation without target bias and without lens (beam of $5 \mathrm{keV}$ directly from source). The second test consists of an implantation with target bias but without lens (beam of $15 \mathrm{keV}$ and target bias at $10 \mathrm{kV}$ ). The third test consists of an implantation with target bias and lens (beam of $15 \mathrm{keV}$, target bias at $10 \mathrm{kV}$ at lens bias at $15.5 \mathrm{kV}$ ).

Figure 8 shows the obtained values. The first test shows that it is not possible to have implantation homogeneity without deceleration, a beam with initial energy of $5 \mathrm{keV}$ suffers too much dispersion along the beam line [11]-[13]. Second test, with deceleration but without electrostatic lens, shows that, as predicted by simulation, implantation fluence is higher in the sample located far from the target edge. 


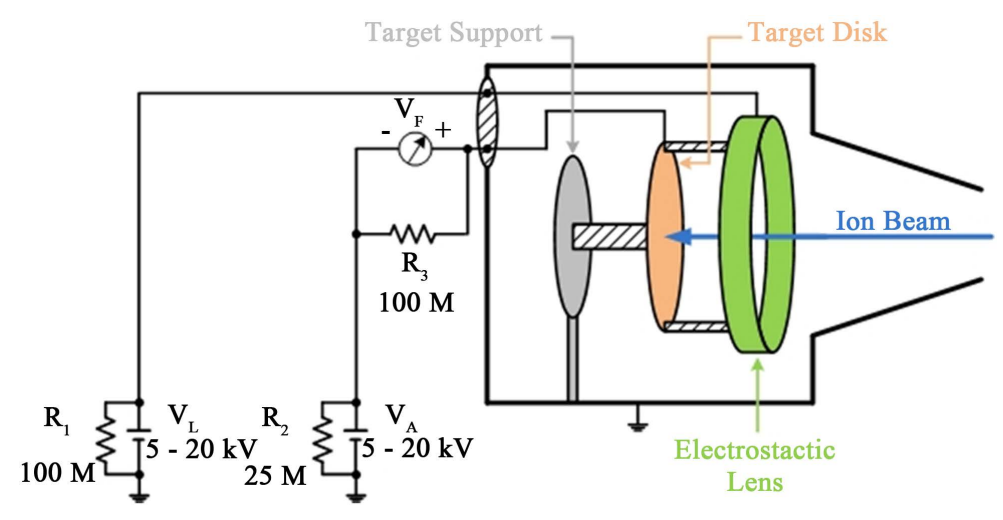

Figure 6. System developed [10].

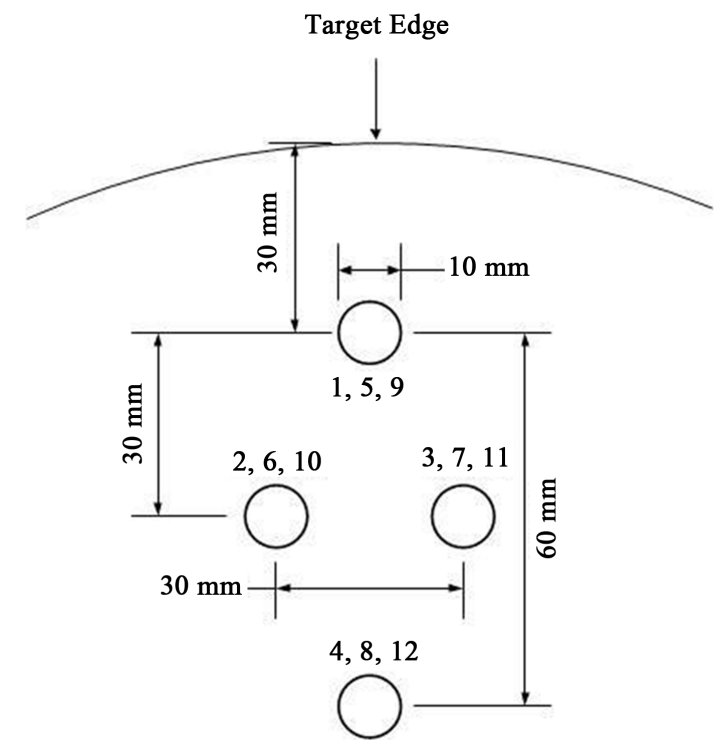

Figure 7. Position arrangement of the silicon samples.

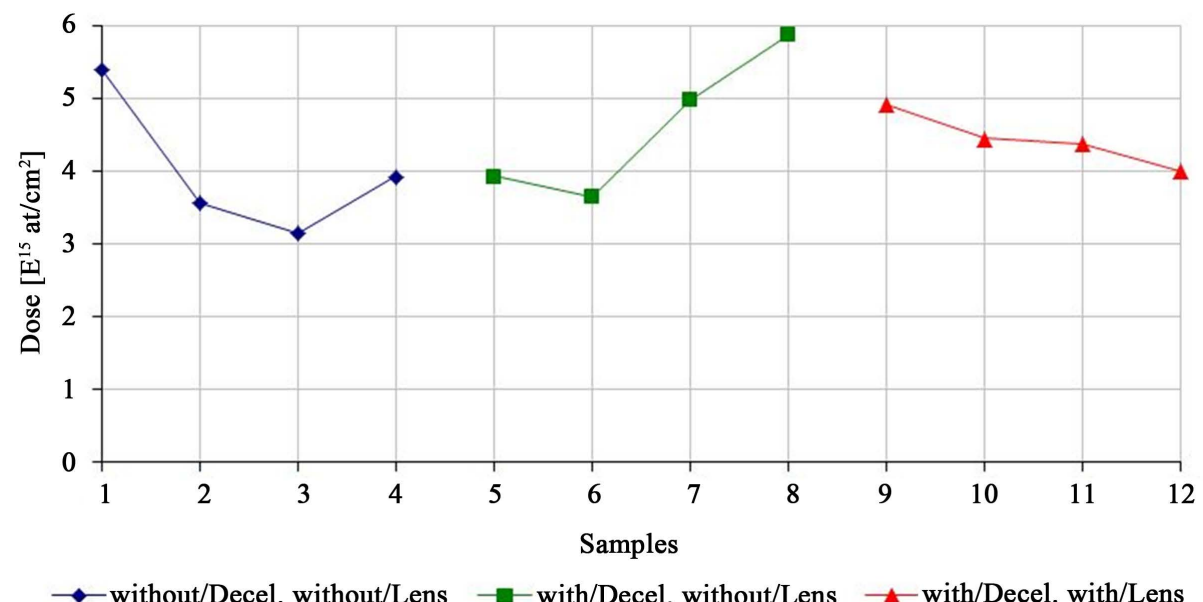

Figure 8. Comparing fluences implanted in the different samples.

Only the third test, with deceleration and lens, shows homogeneity. Notice that, again as predicted by simulation, the fluence is a bit higher for the outmost samples due to beam over-deflection. With this experimental re- 
sult it was observed that there is a greater homogeneity in the samples 9 to 12 with the largest difference between fluences of the order of $14 \%$ while in samples 1 to 4 and 5 to 8 are about $42 \%$ and $38 \%$ respectively.

Through this test, and taking into account the theory the simulation and the results, it is possible to confirm that the maximum limit that each sample can be within the edge of the target is about $6 \mathrm{~cm}$ (sweeping amplitude of $16 \mathrm{~cm}$ ). This can be verified in Figure 7 where sample 9 is distant $3 \mathrm{~cm}$ from the edge, which clearly overcomes the limitations of the system. This test also permits to verify that the trajectory correction due to the use of the lens is concentrated in the center of the target in an area of about $8 \mathrm{~cm}$ in radius, which allows implantations of about $15 \times 15 \mathrm{~cm}^{2}$ with acceptable homogeneity.

\section{Conclusions}

Using the developed system was possible to perform implantations at $5 \mathrm{keV}$ energy continuously scanning of the ion beam over an area of $10 \times 10 \mathrm{~cm}^{2}$ with accuracy for a general purpose low-energy implantation system. In addition it was possible to compare the differences between ion implantation based on energy and beam focus.

In this paper the results obtained for implantations at low energies (below $15 \mathrm{keV}$ ) were presented. These results were achieved based on the system developed with an electrostatic lens of a single electrode, allowing performing implantations at low energies with a focused beam using the magnetic beam scanning over a large area, gaining versatility for research involving ion implantation.

The system developed and applied to the CTN implanter can be used in other implanters, for general purpose low energy implantations where a very high homogeneity and/or very high fluence control is not an issue.

\section{References}

[1] Ziegler, J.F. (2006) Ion Implantation: Science and Technology. 6th Edition, Academic Press, Inc., Yorktown Heights, New York.

[2] Kase, M., Kikuchi, Y., Kubo, T., Niwa, H. and Fukuda, T. (1998) Issues of Ultra Shallow Junction Formation Using Sub-1 keV Ion Implantation. 1998 International Conference on Ion Implantation Technology, Proceedings, Vol. 1 Kyoto, 22-26 June 1998, 110-113. http://dx.doi.org/10.1109/IIT.1999.812064

[3] Graf, M.A., Vanderberg, B., Benveniste, V. and Tieger, D.R. (2002) Low Energy Ion Beam Transport. Proceedings of the 14th International Conference on Ion Implantation Technology, New Mexico, 27-27 September 2002, 359-364. http://dx.doi.org/10.1109/IIT.2002.1258015

[4] Abdelrahman, M.M. (2008) Ion Beam Simulation Using a Three-Electrode Diaphragm Einzel Lens System. Journal of Nuclear and Radition Physics, 3, 93-100.

[5] Dahl, D.A. (2000) Simion 3D Version 8.0-Users’s Manual. Idaho National Engineering and Environmental Laboratory, I D 3415.

[6] Sise, O., Ulu, M. and Dogan, M. (2005) Multi-Element Cylindrical Electrostatic Lens Systems for Focusing and Controlling Charged Particles. Nuclear Instruments and Methods in Physics Research Section A: Accelerators, Spectrometers, Detectors and Associated Equipment, 554, 114-131. http://dx.doi.org/10.1016/j.nima.2005.08.068

[7] Purcell, E.M. (1973) Curso de Física de Berkeley Volume 2. Editora Edgard Blucher 1973.

[8] D. Halliday, D., Resnick, R. and Krane, K.S. (1995) Fundamentos de Física. Livros Técnicos e Científicos.

[9] Hong, S.N., Ruggles, G.A., Paulos, J.J., Wortman J.J. and Ozturk, M.C. (1988) Formation of Ultrashallow p ${ }^{+}$-n Junctions by Low-Energy Boron Implantation Using a Modified Ion Implanter. Applied Physics Letters, 53, 1741-1743. http://dx.doi.org/10.1063/1.100470

[10] Lopes, J.G., Rocha, J., Redondo, L.M. and Alegria, F.C. (2011) High Resolution Ion Beam Profile Measurement System. 13th International Conference on Accelerator and Large Experimental Physics Control Systems - Proceedings, 10-14 October 2011, Grenoble, 164-167.

[11] Shimizu, S., Sasaki, N., Ogata, S. and Tsukakoshi, O. (1996) Ion Beam Deceleration Characteristics of a High-Current, Mass-Separated, Low-Energy Ion Beam Deposition System. Review of Scientific Instruments, 67, 3664-3671. http://dx.doi.org/10.1063/1.1147132

[12] Ranganathan, R., Krull, W., Sundstrom, H. and Mack, M. (1998) Characterization of a High Current Ultra Low Energy Ion Implanter. 1998 International Conference on Ion Implantation Technology Proceedings, Kyoto, 22-26 June 1998, 618-621. http://dx.doi.org/10.1109/IIT.1999.812192

[13] Fukaya, T., Hara, S., Tanaka, Y., Matsumoto, S., Suzuki, T., Fuse, G., et al. (2008) Formation of Ultra-Shallow Junction with $\sim 10 \mathrm{~nm}$ in Si Combined with Low Temperature and Laser Annealing. The 5th International Symposium on 
Advanced Science and Technology of Silicon Materials (JSPS Si Symposium), 10-14 November 2008, Kona, Hawaii. http://www.riam.kyushu-u.ac.jp/nano/hawaii2008/index.html

http://www.researchgate.net/publication/240632078_Formation_of_Ultra-shallow_Junction_with_\%2A10_nm_in_Si Combined with Low Temperature and Laser Annealing 